\title{
RESISTENNCIA A ANTIMICROBIANOS EM CEPAS DE Staphylococcus aureus ISOLADAS DA UTI DE UM HOSPITAL DE CACHOEIRO DE ITAPEMIRIM - ES
}

\section{Ana Carolina Ambrósio Simões ${ }^{1}$ Maicon Marvila Miranda ${ }^{2}$ Camilla Dellatorre Teixeira ${ }^{3}$}

Resumo: A Unidade de Terapia Intensiva, local onde são internados pacientes com maior gravidade, é uma das unidades do hospital com maior índice de infecções hospitalares. Sthapylococcus aureus é um dos maiores problemas clínicos e epidemiológicos em infecções nosocomiais, principalmente os resistentes à oxacilina (MRSA). Esta pesquisa teve como objetivo identificar a prevalência de cepas de S. aureus na UTI de um hospital em Cachoeiro de Itapemirim-ES, nos anos de 2014 e 2015, e determinar sua multirresistência aos antimicrobianos. Os dados foram coletados através do acervo documental do laboratório de análises clínicas. A maior prevalência da bactéria foi no gênero masculino, com 69,15\% em 2014 e 60,18\% em 2015, em amostras de hemocultura (43,62\%) e aspirado traqueal (35,40\%). Encontrouse resistência a alguns antimicrobianos, principalmente aos $\beta$-lactâmicos como a penicilina $(71,28 \% \mathrm{em}$ 2014 e 88,49\% em 2015). A necessidade de uma maior vigilância no controle de infecção hospitalar com o intuito de minimizar e/ou eliminar as infecções de pacientes internados, principalmente em UTI.

Palavras-chave: Sthapylococcus aureus; MRSA; UTI; Antimicrobianos.

\footnotetext{
1 Discente do Curso Bacharelado em Farmácia do Centro Universitário São Camilo - ES, Brasil. E-mail: acasimoes@yahoo.com.br

2 Mestrando da Faculdade de Medicina da Universidade Federal do Rio de Janeiro/ Departamento de Doenças Infecciosas e Parasitárias, Brasil. E-mail: maiconmiranda@outlook.com.

${ }^{3}$ Orientadora. Farmacêutica. Docente do Centro Universitário São Camilo - ES, Brasil. E-mail: camilladellatorre@saocamilo-es.br.
} 